\title{
2. \\ Recherches sur l'élimination, et sur la théorie des courbes.
}

(Par Mr. A. Cayley de Cambridge.)

En désignant par $U, V, W, \ldots$ des fonctions homogènes des ordres $m, n, p$, etc. et d'un nombre égal de variables respectivement, et en supposant que ces fonctions soient les plus générales possibles, c'est-à-dire que le coefficient de chaque terme soit une lettre indéterminée: on sait que les équations $U=0$, $\boldsymbol{V}=0, \boldsymbol{W}=0, \ldots$ offrent une relation $\Theta=0$, dans laquelle les variables n'entrent plus, et où la fonction $\Theta$, que l'on peut nommer „Résultant complet des éyuations," est homogène et de l'ordre np... par rapport aux coefficients de $\boldsymbol{U}$, de l'ordre $m p \ldots$ par rapport à ceux de $\boldsymbol{V}$, et ainsi de suite, tandis qu'elle n'est pas décomposable en facteurs. Cela posé, supposons que les coefficients de $\boldsymbol{U}, \boldsymbol{V}, \ldots$, au lieu d'être tous indéterminés, soient des fonctions quelconques d'un certain nombre de quantités arbitraires. Substiluant ces valeurs dans la fonctions $\Theta$, cette fonction sera toujours le "Résultant complet" des équations. Mais $\Theta$ peut quelquefois être décomposable en facteurs, dont quelques uns doivent être éliminés. En effet les coefficients de $\boldsymbol{U}, \boldsymbol{V}, \ldots$ peuvent contenir des quantités $\xi, \eta, \ldots$ censées comme variables, et d'autres quantités $\alpha, \beta, \ldots$ qui sont constantes, et il peut s'agir de la relation entre $\xi, \eta, \ldots$ qui est nécessaire pour que les équations $U=0, V=0$ etc. puissent subsister conjointement. Dans ce cas tout facteur $\Lambda$ du résultant complet $\Theta$, qui ne contient pas les coefficients variables $\xi, \eta, \ldots$, doit être rejeté. En supprimant ces facteurs, et exprimant par $\Phi$ le facteur qui reste, cette fonction est alors ce que nous nommerons „Résultant réduit." Cependant les facteurs $\boldsymbol{A}$, proprement dits, ne sont jamais des facteurs étrangers, et ce n'est qu'à cause du point de vue particulier, auquel on a envisagé la question, qu'ils ont été rejetés; d'un autre point de vue ces facteurs auraient pû devenir le $R e^{\prime}-$ sultant réduit. Nous les nommerons donc, à l'exemple de M. Sylvestre, „Facteurs spéciaux."

Pour faire voir tout cela avec plus de clarté, prenons pour exemple le problème suivant de Géométrie analytique. 
„Trouver les équations du système des lignes tirées par un point donné aux points d'intersection de deux courbes données."

Soient $U=0, V=0$ les équations des deux courbes, $\boldsymbol{U}, \boldsymbol{V}$ étant des fonctions homogènes des variables $x, y, z$, des ordres $m$ et $n$ respectivement, ce qui revient à prendre $x: z$ et $y: z$ pour coordonnés d'un point. De même il faut exprimer par $(\alpha, \beta, \gamma)$ le point dont les coordonnés sont $\alpha: \gamma$ et $\beta: \gamma$; et ainsi pour tous cas semblables. Il s'entend, qu'on suppose partout que les coefficients de $\boldsymbol{U}, \boldsymbol{V}$, ou de $\boldsymbol{U}$, restent absolument indéterminés. Représentons par $(\alpha, \beta, \gamma)$ le point donné, et $\operatorname{par}(\xi, \eta, \zeta)$ un point quelconque d'une des lignes dont il s'agit. En éliminant $x, y, z$ entre les équations

1. $\boldsymbol{U}=0, \boldsymbol{V}=0$ et $\boldsymbol{x}(\beta \zeta-\gamma \eta)+\gamma(\gamma \xi-\alpha \zeta)+z(\alpha \eta-\beta \xi)=0$, on obtient l'équation cherchée $\Theta=0$. Ici $\Theta$ est une fonction homogène de l'ordre $m n$ par rapport à $\beta \zeta-\gamma \eta, \gamma \xi-\alpha \zeta$ et $\alpha \eta-\beta \xi$; de l'ordre $u$ par rapport aux coefficients de $\boldsymbol{U}$, et de l'ordre $n$ par rapport à ceux de $\boldsymbol{V}$; de plus cette fonction est décomposable en $m n$ facteurs linéaires par rapport à $\xi, \eta, \zeta$, dont les coefficients sont des fonctions irrationnelles de $\alpha, \beta, \gamma$ et des coefficients de $\boldsymbol{U}$ et $\boldsymbol{V}$ (en effet toute fonction homogène de $\beta \zeta-\gamma \eta$, $\gamma \xi-\alpha \zeta, \alpha \eta-\beta \xi$ est douée de cette propriété, qui subsiste encore en changeant entre eux $\xi, \eta, \zeta$ et $\alpha, \beta, \gamma)$. Chaque facteur lineaire, égalé à zéro, appartient à une des lignes en question. Voilà pourquoi $\Theta=0$ est considérée comme équation du système des lignes.

Soit maintenant proposé le problème:

„Trouver l'équation du système des tangentes tirées d'un point fixe à une courbe donnée."

Il y a ici à éliminer $x, y, z$ entre les équations

$$
\text { 2. }\left\{\begin{array}{l}
U=0, \\
\alpha \cdot \frac{d U}{d x}+\beta \cdot \frac{d U}{d y}+\gamma \cdot \frac{d U}{d z}=0 \text { et } \\
x(\beta \zeta-\gamma \eta)+y(\gamma \xi-\alpha \zeta)+z(\alpha \eta-\beta \xi)=0 .
\end{array}\right.
$$

Le résultant complet est une fonction homogène de l'ordre $n(n-1)$ par rapport à $\beta \zeta-\gamma \eta, \gamma \xi-\alpha \zeta$ et $\alpha \eta-\beta \xi$ [n représente ici l'ordre de la fonction $U$ ], de l'ordre $n$ par rapport à $\alpha, \beta, \gamma$, et de l'ordre $2 n-1$ par rapport aux coefficients de $\boldsymbol{U}$. Mais il existe dans ce cas un facteur spécial $\boldsymbol{U}_{0}$ qui est ce que devient $U$ en écrivant $\alpha, \beta, \gamma$ à la place de $x, y, z$. En le mettant de côté, le résultant réduit $\Phi$ est fonction de l'ordre $n(n-1)$ par rapport à 
$\beta \zeta-\gamma \eta, \gamma \xi-\alpha \zeta$ et $\alpha \eta-\beta \xi$ et de l'ordre $2(n-1)$ par rapport aux coefficients de $U$, et l'équation $\Phi=0$ correspond au système de tangentes.

En mettant $x, y, z$ à la place de $\beta \zeta-\gamma \eta, \gamma \xi-\alpha \zeta, \alpha \eta-\beta \xi, \Phi$ devient une fonction de l'ordre $n(n-1)$ par rapport à $x, y, z$, et de l'ordre $2(n-1)$, comme elle l'était ci-dessus par rapport aux coefficients de $U$. Nous désignerons cette nouvelle valeur de $\boldsymbol{\Phi}$. par $F \boldsymbol{U}$; c'est-à-dire nous représenterons par $F \boldsymbol{U}$ le résultant réduit des équations

$$
\text { 3. }\left\{\begin{array}{l}
U=0, \\
\alpha \cdot \frac{d U}{d x}+\beta \cdot \frac{d U}{d y}+\gamma \cdot \frac{d U}{d z}=0 \text { et } \\
x \mathrm{x}+y \mathrm{y}+\mathrm{zz}=0,
\end{array}\right.
$$

FU étant une fonction des ordres $n(n-1)$ et $2(n-1)$ par rapport à x, y, z et aux coefficients de $\boldsymbol{U}$. On sait que l'équation $\boldsymbol{F} \boldsymbol{U}=0$ est celle de la polaire réciproqué de la courbe, par rapport à la conique auxiliaire $x^{2}+y^{2}+z^{2}=0$.

Or les équations (2.) peuvent être écrites aussi sous la forme

$$
\text { 4. }\left\{\begin{array}{l}
U=0, \\
\alpha \cdot \frac{d U}{d x}+\beta \cdot \frac{d U}{d y^{\prime}}+\gamma \cdot \frac{d U}{d z}=0, \\
\xi \cdot \frac{d U}{d x}+\eta \cdot \frac{d U}{d y}+\zeta \cdot \frac{d U}{d z}=0 .
\end{array}\right.
$$

Ici le résultant complet est de l'ordre $n(n-1)$ par rapport à $\alpha, \beta, \gamma$, ou à $\xi, \eta, \zeta$ (car ce résultant complet doit être comme ci-dessus fonction de ce même ordre de $\beta \zeta-\gamma \eta, \gamma \xi-\alpha \zeta$ et $\alpha \eta-\beta \xi)$ et de l'ordre $(n-1)(3 n-1)$ par rapport aux coefficients de $\boldsymbol{U}$. Le facteur spécial dans ce cas est donc une fonction de l'ordre $3(n-1)^{2}$ des coefficients de $\boldsymbol{U}$, et il est facile de trouver sa forme; car on satisferait aux équations (4.) en posant

$$
\frac{d U}{d x}=0, \quad \frac{d U}{d y}=0, \quad \frac{d U}{d z}=0 .
$$

Le résultant de ces équations, que nous désignerons toujours par $K . U$, doit donc se présenter comme facteur spécial du résultant complet du système. Mais K.U étant une fonction des coefficients de l'ordre $3(n-1)^{2}$, elle est précisément le facteur spécial dont il s'agit. (Il est clair que l'équation $\boldsymbol{K}$. $\boldsymbol{U}=\mathbf{0}$ serait la condition nécessitire, pour que la courbe pût avoir un point multiple.)

Reprenons le premier système. On satisfait à la dernière équation en écrivant 6. $\quad x=\alpha l+\xi m, \quad y=\beta l+\eta m$ et $z=\gamma l+\zeta m$.

Soient $[\boldsymbol{U}],[\boldsymbol{V}]$ ce que deviennent $\boldsymbol{U}, \boldsymbol{V}$ par celte substitution. En élimi- 
nant $l, m$ entre les deux équations

$$
[\boldsymbol{U}]=0, \quad[\boldsymbol{V}]=0,
$$

on obtiendra le même résultant $\Theta$ que ci-dessus. En effet, le résultant de ces deux équations est des ordres $n$ et $m$ par rapport aux coefficients de $\boldsymbol{U}$ et $\boldsymbol{V}$, et de l'ordre $2 m n$ par rapport à $\alpha, \beta, \gamma, \xi, \eta$ et $\zeta$ : donc il faut qu'il soit égal à $\Theta$, à un facteur numérique près. On a donc le théorème suivant:

Théorème I. L'équation du système des droites menées par un point donné $(\alpha, \beta, \gamma)$ aux points d'intersection des deux courbes $\boldsymbol{U}=\mathbf{0}, \boldsymbol{V}=\mathbf{0}$, se trouve en éliminant les nouvelles variables $l$, m entre les deux équations $[\boldsymbol{U}]=0$ et $[\boldsymbol{V}]=0$, où $[\boldsymbol{U}],[\boldsymbol{V}]$ sont ce que deviennent $\boldsymbol{U}$ et $\boldsymbol{V}$ par les substitutions $x=\alpha l+\xi m, y=\beta l+\eta m$ et $z=\gamma l+\zeta m$.

En opérant également sur le second système d'équations, on obtient directement le résultant réduit, sans que l'opération fût embarrassée par aucun facteur spécial. En effet, on peut remplacer le système par

$$
\text { 8. }\left\{\begin{array}{l}
\alpha \cdot \frac{d U}{d x}+\beta \cdot \frac{d U}{d y}+\gamma \cdot \frac{d U}{d z}=0, \\
\xi \cdot \frac{d U}{d x}+\eta \cdot \frac{d U}{d y}+\zeta \cdot \frac{d U}{d z}=0 \text { et } \\
x(\beta \zeta-\gamma \eta)+y(\gamma \xi-\alpha \zeta)+z(\alpha \eta-\beta \xi)=0,
\end{array}\right.
$$

et en fesant les substitutions (6.) dans la dernière équation, les deux autres équations se changent en

$$
\frac{d[U]}{d l}=0 \quad \text { et } \quad \frac{d[U]}{d m}=0
$$

où $[\boldsymbol{U}]$ est ce que devient $\boldsymbol{U}$ par cette substitution. Le résultant de ces équations est de l'ordre $2 n(n-1)$ par rapport à $\alpha, \beta, \gamma, \xi, \eta$ et $\zeta$ (c'est-à-dire à une fonction de $\beta \zeta-\gamma \eta, \gamma \xi-\alpha \zeta$ et $\alpha \eta-\beta \xi$, de l'ordre $n(n-1)$ ), et de l'ordre $2(n-1)$ par rapport aux coefficients de $U$; donc il n'y a plus de facteur spécial. Delà suit:

Théorème II. L'équation du système de tangentes menées du point donné $(\alpha, \beta, \gamma)$ à la courbe $U=0$, se trouve en éliminant $l, m$ entre les équations $\frac{d[U]}{d l}=0, \frac{d[V]}{d l}=0,[U]$ étant ce que devient $U$ par les subsjitutions $x=\alpha l+\xi m, y=\beta l+\eta m$ et $z=\gamma l+\zeta m$. En représentant l'équation par $\Phi=0, \Phi$ est une fonction de $\beta \zeta-\gamma \eta, \gamma \xi-\alpha \zeta$ et $\alpha \eta-\beta \xi$, et en remplaçant ces fonctions par $\mathrm{x}, \mathrm{y}, \mathrm{z}$, on obtient l'équation de la polaire réciproque (par rapport à $x^{2}+y^{2}+z^{2}=0$ ) de la courbe donnée.

Crelle's Journal f. J. M. Bd. XXXIV. Heft 1. 
Ce beau théorème est dù à M. Joachimsthal, qui me l'a communiqué l'été passé pendant mon séjours à Berlin, avec démonstration.

On déduit delà, comme cas très-particulier, une formé du résultant des deux équations $a x^{2}+2 b x y+c y^{2}=0$ et $a^{\prime} x^{2}+2 b x y+c^{\prime} y^{2}=0$, citèe dans mon mémoire sur les hyperdéterminants (tome 30 de ce journal). En effet, soit $x^{2}=\mathrm{z}, x y=-\mathrm{y}, y^{2}=\mathrm{x}$ et $\boldsymbol{U}=\mathrm{xz}-\mathrm{y}^{2}$, on aura évidemment $U=0$, $a \cdot \frac{d U}{d \mathrm{x}}+b \cdot \frac{d U}{d \mathrm{y}}+c \cdot \frac{d U}{d \mathrm{z}}=0$ et $a^{\prime} \cdot \frac{d U}{d \mathrm{x}}+\boldsymbol{b}^{\prime} \cdot \frac{d U}{d \mathrm{y}}+\boldsymbol{c}^{\prime} \cdot \frac{d U}{d \mathrm{z}}=0$, donc en cherchant le résultant de ces équations de la manière indiquée dans le théorème, on obtient la formule en question $4\left(a c-b^{2}\right)\left(a^{\prime} c^{\prime}-b^{\prime 2}\right)-\left(a c^{\prime}+a^{\prime} c-2 b b^{\prime}\right)^{2}=0$. Cependant la véritable généralisation de cette formule, à ce que je crois, reste encore à trouver.

Il suit des principes développés dans le mémoire cité, que le résultant des deux équations $\boldsymbol{L}=0, \boldsymbol{M}=\mathbf{0}$ (ò̀ $\boldsymbol{L}, \boldsymbol{M}$ sont des fonctions homogènes des deux variables $l, m$ ) peut toujours être présenté sous la forme $\Theta=\nabla \boldsymbol{L} L$..MM.., où $\nabla$ est une composition d'expressions symboliques, telles que $(12)^{\alpha}(13)^{\beta} \ldots$, dans lesquelles (12) $=\partial_{l_{1}} \partial_{m_{2}}-\partial_{m_{1}} \partial_{l_{2}}$ etc. Par exemple pour $\boldsymbol{L}=a l^{2}+2 b l m+c m^{2}$, $M=a^{\prime} l^{2}+2 b^{\prime} l m+c^{\prime} m^{2}$, on a $\Theta=\left((12)^{2} \cdot(34)^{2}-(13)^{2} \cdot(24)^{2}\right) \cdot \boldsymbol{L} \boldsymbol{L} \cdot \boldsymbol{M} M$ (c'està-dire, comme ci-dessus, $\left.\Theta=4\left(a c-b^{2}\right)\left(a^{\prime} c^{\prime}-b^{\prime 2}\right)-\left(a c^{\prime}+a^{\prime} c-2 b b^{\prime}\right)^{2}\right)$. En appliquant cette théorie à l'élimination des inconnues entre les équations $[U]=0$, $[V]=0$, on oblient

$$
\partial_{l}=\alpha \partial_{x}+\beta \partial_{y}+\gamma \partial_{z} \text { et } \partial_{m}=\xi \partial_{x}+\eta \partial_{y}+\zeta \partial_{z},
$$

et en fésant

$$
\begin{gathered}
\beta \zeta-\gamma \eta=\mathrm{x}, \quad \gamma \xi-\alpha \zeta=\mathrm{y}, \quad \alpha \eta-\beta \xi=\mathrm{z} \text { et } \\
\partial_{y_{1}} \partial_{z_{2}}-\partial_{z_{2}} \partial_{y_{1}}=(12)^{\prime}, \quad \partial_{z_{1}} \partial_{x_{2}}-\partial_{x_{2}} \partial_{z_{1}}=(12)^{\prime \prime}, \quad \partial_{x_{1}} \partial_{y_{2}}-\partial_{x_{2}} \partial_{y_{1}}=(12)^{\prime \prime \prime}, \\
\text { et cela donne } \\
(12)=\mathrm{x}(12)^{\prime}+\mathrm{y}(12)^{\prime \prime}+\mathrm{z}(12)^{\prime \prime \prime}:
\end{gathered}
$$

équations qui peuvent être présentées sous la forme abrégée

$$
(12)=(P 12)
$$

On obtient le résultant cherché en fésant cette substitution dans tous les symboles que contient $\nabla$, et en introduisant $\boldsymbol{U}, \boldsymbol{V}$ au lieu de $[\boldsymbol{U}],[\boldsymbol{V}]$.

Les mêmes remarques peuvent être appliquées au cas d'une élimination entre les deux équations $\frac{d L}{d l}=0, \frac{d L}{d m}=0$, car le résultant sera exprimé ici sous la même forme $\Theta \nabla \boldsymbol{L} \boldsymbol{L}$.. Cherchons par exemple l'équation de la polaire réciproque d'une courbe du second ordre. En observant que le ré- 
sultant des équations $\frac{d L}{d l}=0, \frac{d L}{d m}=0$ (où $L$ est une fonction du second degré) sera exprimé sous la forme $\Theta=(12)^{2} \cdot \boldsymbol{L} \boldsymbol{L}$, on obtient immédiatement pour la reciproque de la courbe du second ordre $\boldsymbol{U}=0$, l'équation

$$
\mathrm{F} \boldsymbol{U}=(\boldsymbol{P} 12)^{2} \cdot \boldsymbol{U} \cdot \boldsymbol{U}=0,
$$

laquelle se réduit en effet à la forme connue.

Passons au cas d'une courbe du troisième ordre. Comme pour une fonction de 'deux variables, le résultant des équations $\frac{d L}{d l}=0, \frac{d L}{d m}=0$ aura la forme $\Theta=(12)^{2} \cdot(34)^{2} \cdot(13) \cdot(42) \cdot L L L E$, on a pour la polaire de $U=0$ : $-2 F \boldsymbol{U}=(\boldsymbol{P} 12)^{2} \cdot(\boldsymbol{P} 34)^{2} \cdot(\boldsymbol{P} 13) \cdot(\boldsymbol{P} 42) \cdot \boldsymbol{U U} \boldsymbol{U} \boldsymbol{U}=0$,

et il serait facile de calculer par là le coefficient d'une puissance ou d'un produit quelconque des variables. Par exemple le coefficient de $z^{6}$ se réduit à $(12)^{2 \prime \prime \prime} \cdot(34)^{2 \prime \prime \prime} \cdot(13)^{2 \prime \prime \prime} \cdot(42)^{\prime \prime \prime} \cdot \boldsymbol{U} \boldsymbol{U} \boldsymbol{U} \boldsymbol{U}$, ou, toute réduction faite, et en supposant $6 U=a x^{3}+b y^{3}+c z^{3}+3 i y^{2} z+3 j z^{2} x+3 k x^{2} y+3 i_{1} y z^{2}+3 j_{1} z x^{2}+3 k_{1} x y^{2}$ $+6 l x y z$

a: $2\left(6 b c i i_{1}-4 i^{3} c-4 i_{1}^{3} b+3 i^{2} i_{1}^{2}-b^{2} c^{2}\right)$. L'équation complètement développée, que j'ai donnée pour cette polaire dans le Cambridge et Dublin Mathematical Journal t. I. p. 97, et que j'ai obtenue par une élimination directe, pourra ainsi être vérifiée.

Nous allons passer maintenant à la théorie des points d'inflexion et des tangentes doubles de la courbe $\boldsymbol{U}=0$. Ces singularités peuvent être traitées par une analyse semblable, en remarquant que parmi les $n(n-1)$ tangentes, menées à la courbe d'un point $\boldsymbol{P}$ situé sur la courbe, la tangente en ce point se présente généralement deux fois, et trois fois, si le point $\boldsymbol{P}$ est un point d'inflexion, ou un point de contact d'une tangente double.

Désignons comme ci-dessus par $(\boldsymbol{U})$ ce que devient $\boldsymbol{U}$ en écrivant $l x+m \xi, l y+m \eta$ et $l z+m \zeta$ à la place de $x, y$ et $z$. En mettant $\xi \partial_{x}+\eta \partial_{y}+\zeta \partial_{z}=\partial$, on a évidemment

$$
[\boldsymbol{U}]=l^{n} \cdot \boldsymbol{U}+\boldsymbol{l}^{n-1} \boldsymbol{m} \cdot \partial \boldsymbol{U}+\frac{1}{1 \cdot 2} l^{n-2} \boldsymbol{n}^{2} \cdot \partial^{2} \boldsymbol{U}+\ldots ;
$$

et en éliminant $l, m$ entre $\frac{d[U]}{d l}=0, \frac{d[U]}{d m}=0$, on obtient un résultant $\Theta=0$, où $\Theta$ est une fonction de $U, \partial U, \partial^{2} U, \ldots \partial^{n} U$, et qui a, comme le remarque M. Joachimsthal, la proprièté dont il s'agit. En écrivant $U=0, \Theta$ contient le facteur $(\partial U)^{2}$; et en meltant de côté ce facteur et écrivant $\partial U=0$, $\Theta$ contient le facteur $\left(\partial^{2} U\right)^{2}$; et ainsi de suite. Nous avons supposé que le 
point $\boldsymbol{P}$, auquel appartiennent les coordonnées $x, y$ et $z$, est un point de la courbe; de manière, que l'on a actuellement $U=0$. En fésant donc cette supposition et en éliminant le facteur $(\partial \boldsymbol{U})^{2}$, l'équation $\Theta=0$ prend la forme $\boldsymbol{X} \partial \boldsymbol{U}+\boldsymbol{Y}\left(\partial^{2} \boldsymbol{U}\right)^{2}=0$ (puisqu'en meltant $\partial \boldsymbol{U}=0$, l'équation contiendra à gauche le facteur $\left.\left(\partial^{2} \boldsymbol{U}\right)^{2}\right)$. Dans le cas où $\boldsymbol{P}$ est un point d'inflexion, ou un point de contact d'une tangente double, cette équation contiendra $\partial \boldsymbol{U}$ comme facteur: donc il faut que $\boldsymbol{Y}\left(\hat{\partial}^{2} \boldsymbol{U}\right)^{2}$ contienne ce facteur, c'est-à-dire: ou $\partial^{2} \boldsymbol{U}$, ou $\boldsymbol{Y}$, contiendra le facteur $\partial \boldsymbol{U}$. Dans le premier cas il s'agit d'un point d'inflexion, dans le sêcond cas d'un point de contact d'une tangente double.

Considérons d'abord les points d'inflexion. Comme $\partial^{2} \boldsymbol{U}$ contient $\partial \boldsymbol{U}$ comme facteur, il faut que cette fonction devienne zéro pour toutes les valeurs

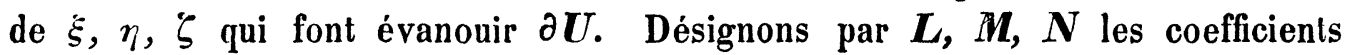
différentiels du premier ordre de $\boldsymbol{U}$, de manière que $\partial \boldsymbol{U}=\boldsymbol{L} \xi+\boldsymbol{M} \eta+\boldsymbol{N} \boldsymbol{\zeta}$. Cette quantité s'évanouit identiquement en fésant $\xi=\beta N-\gamma M, \eta=\gamma L-\alpha N$, $\zeta=\alpha M-\beta L:$ donc il faut que $\partial^{2} \boldsymbol{U}$ s'évanouisse par la substitution de ces valeurs, quelles que soient les quantités $\alpha, \beta, \gamma$. En désignant par $\boldsymbol{D}$ ce que devient le symbole $\boldsymbol{D}$ par cette substitution, c'est-à-dire en fésant

$$
\boldsymbol{D}=\alpha\left(\boldsymbol{M} \partial_{x}-\boldsymbol{N} \partial_{y}\right)+\beta\left(\boldsymbol{N} \partial_{x}-\boldsymbol{L} \partial_{z}\right)+\gamma\left(\boldsymbol{L} \partial_{y}-\boldsymbol{M} \partial_{x}\right),
$$

la condition d'un point d'inflexion se réduit tout simplement à

$$
\boldsymbol{D}^{2} \boldsymbol{U}=0 \text { : }
$$

équation dans laquelle les symboles $\partial_{x}, \partial_{y}, \partial_{z}$ ne doivent pas contenir $\boldsymbol{L}, \boldsymbol{M}, \boldsymbol{N}$. Nous reviendrons sur cette équation dans une note; pour le moment il suffit de remarquer, qu'en vertu de relations qui existent entre $L, M, N$ et les dérivées $a, b, c, f, g, h$ au second ordre, on a identiquement

$$
(n-1)^{2} \cdot D^{2} U=n(n-1) \cdot \Psi \cdot U-(\alpha x+\beta y+\gamma z)^{2}(\nabla U),
$$

où $\Psi$ est une fonction de $\alpha, \beta, \gamma$ et des dérivées $a, b, c, f, g, h$, dont la forme sera donnée dans la suite, et

$$
\nabla \boldsymbol{U}=a b c-a f^{2}-b g^{2}-c h^{2}+2 f g h .
$$

Donc à cause de $U=0$, la seule condition pour déterminer les points d'inflexion est l'équation

$$
\nabla \boldsymbol{U}=\mathbf{0}
$$

qui est de l'ordre $3(n-2)$ par rapport aux variables, et de l'ordre 3 par' rapport aux coefficients de $U$. Cela est déjà connu par les recherches de M. Hesse. J'ai donné ici cette équation pour faire voị la liaison qui existe entre cette question et celle de trouver les tangentes doubles, à laquelle je vais passer maintenant. 
On obtient l'équation, qui détermine les points de contact de ces tangentes, en fésant les mêmes substitutions $\xi=\beta N-\gamma \boldsymbol{M}$ etc. dans la fonction $\boldsymbol{Y}$, et en égalant à zéro les coefficients des différentes puissances ou produits de $\alpha, \beta, \gamma$. Remarquons que cette fonction $\boldsymbol{Y}$ s'obtient par une fonction de l'ordre $n^{2}-n$ par rapport à $x, y, z$, ou à $\xi, \eta, \zeta$, et de l'ordre $2(n-1)$ par rapport aux coefficients, en éliminant les facteurs $(\partial \boldsymbol{U})^{2}$ et $\left(\dot{\sigma}^{2} \boldsymbol{U}\right)^{2}$, qui ensemble montent au degré $4 n-6$ par rapport à $x, y, z$, à 6 par rapport à $\xi, \eta, \zeta$, et à 4 par rapport aux coefficients. Donc $Y$ est des degrés $n^{2}-n-6$, $n^{2}-5 n-6$ et $2 n-6$ par rapport à $x, y, z, \xi, \eta, \zeta$ et aux coefficients de $\boldsymbol{U}$ respeclivement. En substituant donc $\xi, \eta, \zeta$, celte fonction devient des ordres $n^{2}-n-6$ par rapport à $\alpha, \beta, \gamma$, à $n^{3}-2 n^{2}-10 n+12$ par rapport à $x, y, z$ [savoir $\left.\left(n^{2}-5 n-6\right)+(n-1)\left(n^{2}-n-6\right)\right]$, et de l'ordie $n^{2}+n-12$ par rapport aux coefficients [savoir $(2 n-6)+\left(n^{2}-n-6\right)$ ]. Mais on sait que les conditions de l'évanouissement de $\boldsymbol{Y}$ doivent se réduire à une seule équation; et cela ne peut arriver que de la même manière dont la réduction analogue a eu lieu pour les points d'inflexion. En écrivant donc $[\boldsymbol{Y}]$ à la place de ce que devient $\boldsymbol{Y}$ après la substitution, il faut que l'on ait identiquement:

$$
[\boldsymbol{Y}]=\boldsymbol{\Lambda} \cdot \boldsymbol{U}+\boldsymbol{N}(\boldsymbol{\Pi U}) \text {. }
$$

$N$ étant fonction de $\alpha, \beta, \gamma$ du degré $n^{2}-n-6$. Il parait de plus probable que cette fonction aura la même forme que celle pour les points d'inflexion, savoir $\boldsymbol{N}=(\alpha \boldsymbol{x}+\beta y+\gamma z)^{n^{2-n-6}} . \quad(\Pi \boldsymbol{U}$, comme ci-dessus $\nabla \boldsymbol{U}$, est censé exprimer non pas un produit, mais une derivée de $U$ : de même plus bas $\boldsymbol{P U}$ et $\boldsymbol{Q U}$.) Cela étant, $\Pi \boldsymbol{U}$ sera du degré $(n-2)\left(n^{2}-9\right)$ par rapport à $x, y$ et $z$ [c'est-à-dire $\left.n^{3}-2 n^{2}-10 n+12-\left(n^{2}-n-6\right)\right]$, et du degré $n^{2}+n-12$ par rapport aux coefficients. On a donc le théorème suivant:

Théorène. On trouve les points de contact des tangentes doubles, en combinant avec l'équation de la courbe une équation $\Pi U=0$ de l'ordre $(n-2)\left(n^{2}-9\right)$ par rapport aux variables, et de l'ordre $n^{2}+n-12$ par rapport aux coefficients; c'est-à-dire: puisqu'il correspond deux points de contact à une tangente double, le nombre de ces tangentes est égal à $\frac{1}{2} n(n-2)\left(n^{2}-9\right)$ : théorème démontré indirectement par M. Plücker.

Cherchons maintenant les équations du système des tangentes aux points d'inflexion et du système des tangentes doubles.

Pour trouver la première équation, il faut éliminer $x, y, z$ entre les trois équations

$$
U=0, \quad \nabla U=0, \quad \mathrm{x} \cdot \frac{d U}{d x}+\mathrm{y} \cdot \frac{d U}{d y}+\mathrm{z} \cdot \frac{d U}{d z}=0 .
$$


Le résultant complet sera du degré $3 n(n-2)$ par rapport à $x, y$ et $z$, et de l'ordre $9 n^{2}-18 n+6$ [savoir $3(n-2)(n-1)+3 n(n-1)+3 n(n-2)$ ] par rapport aux coefficients; mais il existe ici le facteur spécial $(\boldsymbol{K U})^{2}$, et ce facteur étant éliminé, on obtient un résultant réduit $\boldsymbol{Q U}=0$, du degré $3 n(n-2)$ par rapport aux variables, et du même degré $3 n(n-2)$ par rapport aux coefficients.

De mème on aura l'équation du système des tangentes doubles, en éliminant $x, y$ et $z$ entre les trois équalions

$$
\boldsymbol{U}=0, \quad \Pi U=0 \quad \text { et } \quad \mathrm{x} \cdot \frac{d U}{d x}+\mathrm{y} \cdot \frac{d U}{d y}+\mathrm{z} \cdot \frac{d U}{d z}=0 .
$$

Le résultant complet est ici du degré $n(n-2)\left(n^{2}-9\right)$ par rapport à x, y, z, et du degré $3 n^{4}-5 n^{3}-29 n^{2}+57 n-18$ [savoir $(n-1)(n-2)\left(n^{2}-9\right)$ $\left.+n(n-2)\left(n^{2}-9\right)+n(n-1)\left(n^{2}+n-12\right)\right]$ par rapport aux coefficients. Mais il existe de même dans ce cas un facteur spécial $(\boldsymbol{K U})^{n^{2-n-6}} \dot{[}$ du degré $\left.3(n-1)^{2} \cdot\left(n^{2}-n-6\right)=3 n^{4}-9 n^{3}-9 n^{2}+33 n-18\right]$, et en l'éliminant, le résultant réduit sera du degré $4 n(n-2)(n-3)$ par rapport aux coefficients. Mais le terme de cette équation à gauche sera évidemment un carré; on aura donc pour l'équation du système des tangentes doubles, la relation $\boldsymbol{P U}=0$, où $\boldsymbol{P U}$ est une fonction du degré $\frac{1}{2} n(n-2)\left(n^{2}-9\right)$ par rapport aux variables, el du degré $2 n(n-2)(n-3)$ par rapport aux coefficients. Donc on pourra former le tableau suivant des degrés des différentes équations obtenues:

Degrés par rapport

Equation de la courbe $\boldsymbol{U}=0, \ldots \ldots \ldots$

\begin{tabular}{c|c}
\hline aux variables. & aux coefficients. \\
$n$ & 1 \\
0 & $3(n-1)^{2}$ \\
$n(n-1)$ & $2(n-1)$ \\
$3 n(n-2)$ & 3 \\
$(n-2)\left(n^{2}-9\right)$ & $(n+4)(n-3)$ \\
$3 n(n-2)$ & $3 n(n-2)$ \\
& \\
& \\
& \\
& \\
&
\end{tabular}

Condition d'un point multiple $\boldsymbol{K} \boldsymbol{U}=\mathbf{0}, \ldots$

Polaire réciproque $\mathrm{FU}=0, \ldots \ldots \ldots(n-1)$

Courbe des inflexions $\nabla \boldsymbol{U}=0, \ldots \ldots 3 n(n-2)$

Courbes des contacls des tangentes doubles $\boldsymbol{\Pi} \boldsymbol{U}=0, \ldots \ldots \ldots \ldots$

Systèmes des tangentes aux points d'inflexion $\boldsymbol{Q U}=0, \ldots \ldots \ldots \ldots \ldots \ldots \ldots \ldots(n-2)$

Système des langentes doubles $\boldsymbol{P} U=0, \ldots \frac{1}{2} n(n-2)\left(n^{2}-9\right)\left(2 n^{\prime}(n-2)(n-3)\right.$.

La polaire de la polaire réciproque $F F U$ sera évidemment du degré $\left(n^{2}-n\right)\left(n^{2}-n-1\right)$ par rapport aux variables, et du degré $4(n-1)\left(n^{2}-n-1\right)$ par rapport aux coefficients. Cette polaire de la polaire contiendra, comme on le sait par la théorie géometrique développée par M. Plücker, les facteurs 
$\boldsymbol{U},(\boldsymbol{P U})^{2}$ et $(\boldsymbol{Q U})^{3}$; il faut y ajouter encore le facteur constant $\boldsymbol{K} \boldsymbol{U}$, et l'on aura definitivement l'équation

$$
\mathrm{FF} \boldsymbol{U}=(\boldsymbol{K} \boldsymbol{U})(\boldsymbol{P} \boldsymbol{U})^{2}(\boldsymbol{Q} \boldsymbol{U})^{3} \cdot \boldsymbol{U}
$$

dans laquelle il sera facile à vérifier que les deux côtés sont des mêmes degrés par rapport aux variables et aux constantes. En effet on a

$$
\begin{aligned}
& 4(n-1)\left(n^{2}-n-1\right)=3(n-1)^{2}+4 n(n-2)(n-3)+9 n(n-2)+1 \text { et } \\
& n(n-1)\left(n^{2}-n-1\right)=n(n-2)\left(n^{2}-9\right)+9 n(n-2)+9 .
\end{aligned}
$$

Mon but a été ici de donner une idée précise des theorèmes à démontrer, pour former une théorie toute analylique des polaires réciproques; je n'ai fait qu'avancer ces theorèmes (sans chercher à les démontrer), pour faire voir leur liaison avec la théorie de l'élimination et avec celle des hyperdéterminants; c'est à cette dernière en particulier qu'il faut, je crois, recourir pour démontrer la formule donnée ci-dessus $[\boldsymbol{Y}]=\Lambda \cdot \boldsymbol{U}+(\alpha \boldsymbol{x}+\beta y+\gamma \boldsymbol{z})^{n^{2}-n-6}(\boldsymbol{\Pi} \boldsymbol{U})$, et pour trouver définitivement la forme de la dérivée $\boldsymbol{\Pi U}$, au moyen de laquelle on déterminera les points de contact des tangentes doubles. Je serais bien aise que, ces recherches puissent de quelque manière faciliter la solution du problème des réciproques des surfaces: objet, qui est resté encore dans une complète obscurité.

\section{Note sur les points d'inflexion.}

Je vais d'abord rassembler plusieurs formules qui se rapportent au système des coefficients dans le développement de $D^{2} U$. On a

$$
D^{2} U=a \alpha^{2}+\mathrm{b} \beta^{2}+c \gamma^{2}+2 \mathfrak{f} \beta \gamma+2 g \gamma \alpha+2 h \alpha \beta,
$$

où

$$
\begin{aligned}
& \mathbf{a}=M^{2} c-2 M N f+N^{2} b, \\
& \mathbf{b}=N^{2} a-2 N L g+L^{2} c, \\
& \mathbf{c}=L^{2} b-2 L M h+M^{2} a, \\
& \mathbf{f}=-M N a+N L h+L M g-L^{2} f, \\
& \mathbf{g}=+M N h-N L b+L M f-M^{2} g, \\
& \mathbf{h}=+M N g+N L f-L M c-N^{2} h .
\end{aligned}
$$

Nous écrirons de plus, pour abréger,

$$
\begin{aligned}
& b c-f^{2}=\mathfrak{A}, \quad g h-a f=\mathfrak{f}, \quad \nabla=a b c-a f^{2}-b g^{2}-c h^{2}+2 f g h, \\
& \boldsymbol{i} \boldsymbol{a}-\boldsymbol{g}^{2}=\mathfrak{g}, \quad h f-b g=\mathfrak{G}, \\
& a b-h^{2}=\mathfrak{C}, \quad f g-c h=\mathfrak{g}, \\
& \text { bc }-\mathbf{f}^{2}=(\mathfrak{X}) \text {, etc. }
\end{aligned}
$$


et enfin

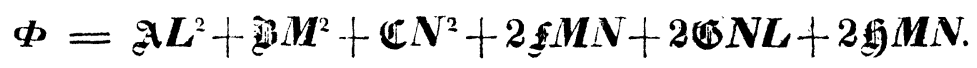

On obtient identiquement

$$
\begin{aligned}
& \boldsymbol{L} \mathbf{a}+\boldsymbol{M} \mathbf{h}+\boldsymbol{N}_{\mathrm{g}}=\mathbf{0}, \\
& \boldsymbol{L} \mathrm{h}+\boldsymbol{M} \mathrm{b}+\boldsymbol{N f}=\mathbf{0}, \\
& \boldsymbol{L} \mathrm{g}+\boldsymbol{M} \mathbf{f}+\boldsymbol{N}_{\mathbf{c}}=\mathbf{0} ; \\
& \text { (ג) }=L^{2} \Phi, \\
& \text { (艘) }=\boldsymbol{M}^{2} \boldsymbol{\Phi} \text {, } \\
& (\mathfrak{C})=\boldsymbol{N}^{2} \boldsymbol{\Phi}, \\
& \text { (ङ) }=\mathbf{M N \Phi} \text {, } \\
& (\mathfrak{G})=\mathbf{N} \boldsymbol{L} \Phi, \\
& \text { (fi) }=\boldsymbol{L} \boldsymbol{M \Phi} \text {; }
\end{aligned}
$$

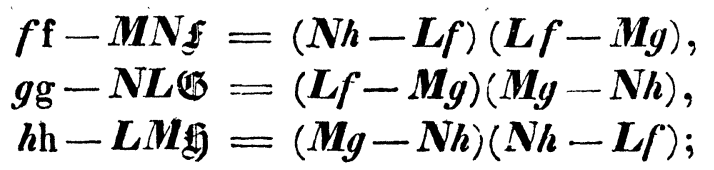

$4 L^{2} M N+b\left(L^{2} b+M^{2} a\right)+c\left(\Lambda^{2} a+L^{2} c\right)-b c-2 L^{2} a a=\left(L^{2} b-M^{2} a\right)\left(N^{2} a+L^{2} c\right)$, $4 M^{2} N L \mathfrak{b}+\mathbf{c}\left(M^{2} c+N^{2} b\right)+a\left(L^{2} b+M^{2} a\right)-c a-2 M^{2} b b=\left(M^{2} c-N^{2} b\right)\left(L^{2} b-M^{2} a\right)$, $4 \boldsymbol{N}^{2} \boldsymbol{L} M \mathfrak{d}+\mathbf{a}\left(\boldsymbol{N}^{2} a+L^{2} c\right)+b\left(M^{2} c+N^{2} b\right)-a b-2 N^{2} c c=\left(N^{2} a-L^{2} c\right)\left(M^{2} c-N^{2} b\right) ;$

$$
\begin{aligned}
& 4 L^{2} M N A-a^{2}+2 a\left(M^{2} c+N^{2} b\right)=-\left(M^{2} c-N^{2} b\right)^{2}, \\
& 4 M^{2} N L-b^{2}+2 b\left(N^{2} a+L^{2} c\right)=-\left(N^{2} a-L^{2} c\right)^{2}, \\
& 4 N^{2} L M U-c^{2}+2 c\left(L^{2} b+M^{2} a\right)=-\left(L^{2} b-M^{2} a\right)^{2} ;
\end{aligned}
$$

$L^{2} M N_{c}+N_{g}(+L f+M g-N h)+M h(L f-M g+N h)-g h=-M N(N h-M g)^{2}$, $M^{2} \mathbf{N} \boldsymbol{L}+\boldsymbol{L h}(-\boldsymbol{L} f+M g+N h)+N \mathrm{f}(\boldsymbol{L} f+M g-N h)-\mathrm{hf}=-\mathbf{N} \boldsymbol{L}(\boldsymbol{L} f-\mathbf{N} \boldsymbol{h})^{2}$, $\boldsymbol{N}^{2} \boldsymbol{L} \boldsymbol{M C}+\boldsymbol{M} \mathbf{f}(+\boldsymbol{L} \boldsymbol{f}-\boldsymbol{M} \boldsymbol{g}+\boldsymbol{N} \boldsymbol{h})+\boldsymbol{L} \mathrm{g}(\boldsymbol{L} \boldsymbol{f}+\boldsymbol{M} \boldsymbol{g}+\boldsymbol{N} \boldsymbol{h})-\mathrm{fg}=-\boldsymbol{L} \boldsymbol{M}(\boldsymbol{M} \boldsymbol{g}-\boldsymbol{L} \boldsymbol{f})^{2} ;$

$$
\begin{gathered}
2 \nabla \boldsymbol{L}^{2}=-a^{2} \mathrm{a}+\left(a b-2 h^{2}\right) \mathrm{b}+\left(c a-2 g^{2}\right) \mathrm{c}+2(a f-2 g h) \mathrm{f}-2 a g \cdot \mathrm{g}-2 a h \cdot \mathrm{h}, \\
2 \nabla M^{2}=+\left(a b-2 h^{2}\right) \mathrm{a}-b^{2} \mathrm{~b}+\left(b c-2 f^{2}\right) \mathrm{c}-2 b f \cdot \mathrm{f}+2(b g-2 h f) \mathrm{g}-2 b h \cdot \mathrm{h}, \\
\left.2 \nabla \boldsymbol{N}^{2}=+\left(c a-2 y^{2}\right) \mathrm{a}+b c-2 f^{2}\right) \mathrm{b}-c^{2} \mathrm{c}-2 c f \cdot \mathrm{f}-2 c g \mathrm{~g}+2(c h-2 f g) \mathrm{h} \\
2 \nabla M N=+(a f-2 g h) \mathrm{a}-b f \mathrm{~b}-c f \mathrm{c}-2 b c \mathrm{f}-2 c h \mathrm{~g}-2 b g \mathrm{~h}, \\
2 \nabla \boldsymbol{N}=-a g \mathrm{a}+(b g-2 h f) \mathrm{b}-c g \mathrm{c}-2 c h \mathrm{f}-2 c a \mathrm{~g}-2 a f \mathrm{~h}, \\
2 \nabla \boldsymbol{L}=-a h \mathrm{a}-b h \mathrm{~b}+(c h-2 f g) \mathrm{c}-2 b g \mathrm{f}-2 a f \mathrm{~g}-2 a b \mathrm{~h} .
\end{gathered}
$$

Dans toutes ces équations, si $L x+M y+N_{\approx}$ était facteur de $a x^{2}+b y^{2}+c z^{2}$ $+2 f y z+2 g z x+2 h x y$, on aurait évidemment $\mathrm{a}=0, \mathrm{~b}=0, \mathrm{c}=0, \mathrm{f}=0$, $\mathrm{g}=0, \mathrm{~h}=0$, et de là $\nabla=0, \Phi$, ce qui donnerait des formules plus simples, et auxquelles on pourrait encore donner plusieurs autres formes. Par exemple on tire d'un de ces systèmes, $-1 \div M N \xi=M g-N / \ell \div \Theta$ etc, où $\Theta=$ $(\boldsymbol{M g}-\boldsymbol{N} \boldsymbol{h})(\boldsymbol{N} \boldsymbol{h}-\boldsymbol{L} \boldsymbol{f})(\boldsymbol{L} \boldsymbol{f}-\boldsymbol{M g})$, et delà les expressions 


$$
\begin{aligned}
& \frac{L}{\boldsymbol{f}}+\frac{\boldsymbol{M}}{\mathfrak{G}}+\frac{\boldsymbol{N}}{\mathfrak{d}}=\mathbf{0}, \\
& \frac{L^{2} f}{£}+\frac{M^{2} g}{\mathbb{E}}+\frac{N^{2} h}{(j)}=0 \text {, } \\
& \frac{L^{3} f^{2}}{f}+\frac{M^{3} g^{2}}{(\mathbb{G}}+\frac{N^{3} h^{3}}{\mathbb{E})}=L M N \text {, }
\end{aligned}
$$

auxquelles on pourrait ajouter encore plusieurs systèmes analogues, ce qui se ferait sans la moindre difficulté.

Jusqu'ici $L, M, N, a, b, c, f, g, h$ ont été des quantités quelconques. En supposant qu'elles soient les dérivées du premier et du second ordre d'une fonction $U$, on a

$$
\begin{aligned}
& (n-1) L=a x+h y+g z \\
& (n-1) M=h x+b y+f z \text {, } \\
& (n-1) \mathbf{N}=g x+f y+c z \text {, } \\
& n \cdot(n-1) U=a x^{2}+b y^{2}+c z^{2}+2 f y z+2 g x z+2 h x y \text {, }
\end{aligned}
$$

et la substitution de ces valeurs donne la formule du texte:

$$
(n-1)^{2} \cdot D^{2} U=n \cdot(n-1) \cdot \Psi \cdot U-(\alpha x+\beta y+\gamma z)^{2}(\nabla \boldsymbol{U}),
$$

$(\nabla \boldsymbol{U})$ étant ici $=\nabla$, et $\Psi$ étant donnée par l'équation

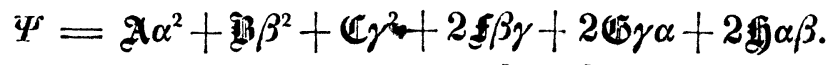

Cela peut être vérifié facilement par la substitution actuelle. Mais nous allons le démontrer par la théorie des hyperdéterminants. En effet, soit (123) le déterminant symbolique formé avec $\partial_{x_{1}}, \partial_{y_{1}}, \partial_{z_{1}}: \partial_{x_{2}}, \partial_{y_{2}}, \partial_{z_{2}}: \partial_{x_{3}}, \partial_{y_{3}}, \partial_{z_{3}}$, (A23) le déterminant symbolique formé avec $\alpha, \beta, \gamma: \partial_{x_{2}}, \partial_{y_{2}}, \partial_{z_{2}}: \partial_{x_{3}}, \partial_{y_{3}}, \partial_{z_{3}}$, et ainsi de suite; puis soient $\boldsymbol{T}_{1}, \boldsymbol{T}_{2} \ldots$ les fonctions $x \partial_{x_{1}}+y \partial_{y_{1}}+z \partial_{z_{1}}$, $x \partial_{x_{2}}+y \partial_{y_{2}}+z \partial_{z_{2}}$ etc. et $\varrho=\alpha x+\beta y+\gamma z$, on aura identiquement:

$$
\varrho \cdot(123)=-T_{1} \cdot(A 23)-T_{2} \cdot(A 31)-T_{3}(A 12),
$$

et en élévant au carré les deux membres de cette équation, ajoutant le facteur $\boldsymbol{U}_{1} \boldsymbol{U}_{2} \boldsymbol{U}_{3}$, et réduisant les variables après avoir différentié suivant $x, y, z$, puis en tenant compte des équations

$$
\begin{aligned}
& (123)^{2} U_{1} U_{2} U_{3}=6 \nabla U, \\
& T_{1}^{2} \cdot A(23)^{2} U_{1} U_{2} U_{3}=2 n(n-1) U \cdot \Psi \text { et } \\
& T_{1} T_{2} A(23) A(31) U_{1} U_{2} U_{3}=-(n-1)^{2} D^{2} U,
\end{aligned}
$$

on obtiendra le résultat dont il s'agit.

Il sera aussi facile de déduire de celte formule quelques propriétés de la courbe $\nabla \boldsymbol{U}=0$, dans les cas d'un point double ou d'un point de rebroussement de la courbe $\boldsymbol{U}=0$. En effet, pour un point double, les dérivées 
$\boldsymbol{L}, \boldsymbol{M}, \boldsymbol{N}$ de $\boldsymbol{U}$, et par suite $\boldsymbol{D}^{2} \boldsymbol{U}$, et ses dérivées du premier ordre, s'évanouissent. De plus, pour un point de rebroussement on a $\Psi=0$. Donc, pour un point double on a $\nabla \boldsymbol{U}=0$, où la courbe exprimée par celte équation passe par chaque point double ( $\mathrm{y}$ compris les points de rebroussement) de la courbe $\boldsymbol{U}=0$. Prenons la dérivée de l'équation en question, en affectant du symbole $\partial=\xi \partial_{x}+\eta \partial_{y}+\zeta \partial_{z}$ ses deux membres. Supprimant les termes, qui se réduisent à zéro aux points doubles, cela donne

$$
0=(\alpha x+\beta y+\gamma z)^{2} \cdot \partial \nabla \boldsymbol{U} ;
$$

c'est-à-dire qu'il y aura aussi à chacun de ces points un point double de la courbe $\nabla \boldsymbol{U}=0$. Passant aux dérivées du second ordre, on aura

$$
(n-1)^{2} \cdot \partial^{2} D^{2} U=n(n-1) \Psi \partial^{2} U-(\alpha x+\beta y+\gamma z)^{2} \cdot \partial^{2} \nabla U
$$

Or ici

$$
\partial^{2} \boldsymbol{D}^{2} \boldsymbol{U}=\alpha^{2} \cdot \partial^{2} \mathrm{a}+\beta^{2} \partial^{2} \mathrm{~b}+\gamma^{2} \cdot \partial^{2} \mathbf{c}+2 \beta \gamma \partial^{2} \mathrm{f}+2 \gamma \alpha \partial^{2} \mathrm{~g}+2 \alpha \beta \partial^{2} \mathrm{~h}
$$

et $\partial^{2} \mathbf{a}$ etc. se réduisent $\dot{a}$

$$
2\left(\partial M^{2} \cdot c-2 \partial M \cdot \partial N f+\partial N^{2} \cdot b\right) \text { etc., }
$$

savoir en mettant $\boldsymbol{a} \xi+l i \dot{\eta}+\boldsymbol{g} \zeta ; \boldsymbol{h} \xi+b \eta+f \zeta, g \xi+f \eta+c \zeta$ a la place de $\partial \boldsymbol{L}$, $\partial \boldsymbol{M}, \partial \boldsymbol{N}$, et en ayant ëgard à la condition $\nabla \boldsymbol{U}=0$, ’̀ $2 \partial^{2} \boldsymbol{U} \cdot \boldsymbol{A}$, etc., on aura $\partial^{2} \boldsymbol{D} u=2 \partial^{2} \boldsymbol{U} \cdot \Psi$ ou enfin

$$
(n-1)(n-2) \partial^{2} \boldsymbol{U} \cdot \Psi=-(\boldsymbol{x}+\boldsymbol{\beta} \boldsymbol{y}+\boldsymbol{\gamma} \boldsymbol{z})^{2} \cdot \partial^{2} \nabla \boldsymbol{U},
$$

c'est-à-dire: les deux courbes $\boldsymbol{U}=0, \nabla \boldsymbol{U}=0$, se toucheront dans les points doubles. Enfin pour un point de rebroussement $\dot{\partial}^{2} \nabla \boldsymbol{U}$ s'évanouit, c'est-à-dire, il existe un point triple dans la courbe $\nabla \boldsymbol{U}=0$. Mais il peut être démontré que deux branches de la courbe se touchent en ce point, et qu'elles touchent aussi la courbe $\boldsymbol{U}=0$; c'est-à-dire qu'il y aura dans la courbe $\nabla \boldsymbol{U}=0$ un point de rebroussement, et une autre branche de la courbe qui passe par ce point. Pour cela il faudra passer aux dérivées du troisième ordre. Cela donne, en supprimant les termes qui s'évanouissent:

$$
(n-1)^{2} \cdot \partial^{3} \cdot \boldsymbol{D}^{2} \boldsymbol{U}=3 \boldsymbol{n}(\boldsymbol{n}-1) \partial \Psi \cdot \partial^{2} \boldsymbol{U}-(\alpha x+\beta y+\gamma \approx)^{2} \cdot \partial^{3} \nabla \boldsymbol{U} .
$$

Ici on a

$$
\begin{gathered}
\partial^{3} D^{2} U=\alpha^{2} \partial^{3} \mathbf{a}+\text { etc. }, \\
\partial^{3} \mathbf{a}=6\left(\partial M^{2}, \partial c-2 \partial M \partial \boldsymbol{N} \partial f+\partial \boldsymbol{N}^{2} \cdot \partial b\right) \\
+6\left(\partial M \partial^{2} M c-\left(\partial M \partial^{2} \boldsymbol{N}+\partial \boldsymbol{N} \partial^{2} \boldsymbol{M}\right) f+\partial \boldsymbol{N} \cdot \partial^{2} \boldsymbol{N} b\right) \text { etc. }
\end{gathered}
$$

et les deux lignes de cette expression se réduisent à $6 \partial^{2} \boldsymbol{U} \cdot \partial \mathfrak{A}$ et à zéro respectivement. En effet, en rémplaçant $\partial M, \partial N$ par leurs valeurs, le coefficient de $\xi^{2}$ dans la prémière ligne devient $6\left(h^{2} \partial c-2 g h \partial f+g^{2} \partial b\right)=$ $6 a(b \partial c+c \partial b(-2 f \partial \dot{f})$ (et cause de $=0, \mathbb{C}=0, f=0)=6 a \partial A$ 
et également pour les autres termes. De même, le coefficient de $\xi^{2}$ dans la seconde ligne devient $6(h \partial h \cdot c-(g \partial h+h \partial g) f+g \partial g \cdot b) . \quad$ En y ajoutant $3\left(h^{2} \partial c-2 g h \partial f+g^{2} \partial b\right)$, savoir $3 a \partial \mathcal{A}$, la somme se réduit à $3 \partial\left(c h^{2}-2 f g h+b g^{2}\right)$ $=3 \partial\left(a_{\mathfrak{A}} \mathfrak{A}-\nabla\right)=3 a \partial \mathfrak{A}$; donc le coefficient en question s'évanouit. En cherchant de la même manière les autres coefficients, on trouvera les valeurs dont il s'agit, et ainsi $\partial^{3} \mathbf{a}=6 \partial^{2} \boldsymbol{U} \cdot \partial \boldsymbol{A}$ etc. et delà $\partial^{3} \boldsymbol{D}^{2} \boldsymbol{U}=6 \partial^{2} \boldsymbol{U} \partial \Psi$; donc enfin

$$
\left.3(n-1)(n-2) \cdot \partial^{2} U \partial \Psi=-(\alpha x+\beta) ;+\gamma \approx\right)^{2} \cdot \partial^{3} \nabla \boldsymbol{U} ;
$$

ce qui suffit pour démontrer le théorème, qui peut être énoncé comme suit:

Théorème. "Il existe un point double de la courbe $\nabla \boldsymbol{U}=0$, pour chaque point double de la courbe $\boldsymbol{U}=0$, et les deux courbes ont des tangentes communes dans ces points. De plus, il existe un point triple de la courbe $\nabla \boldsymbol{U}=0$, pour chaque point de rebroussement de la courbe $\boldsymbol{U}=0$, savoir un point de rebroussement dont les deux branches touchent la tangente de la courbe $\boldsymbol{U}=0$, et encore une troisième branche, qui passe par le point de rebroussement."

Il suit de là que dans le cas d'un point double, ce point dòit être considéré comme la réunion de six points d'intersection, et dans celui d'un point de rebroussement, de huit points d'intersection. C'est de celte manière que l'on se rend compte du théorème de M. Plücker qui dit que l'effet de ces deux singularités est, de faire disparaitre respectivement six ou huit points d'inflexion de la courbe donnée.

Examinons, en finissant, la théorie des points d'osculation. Il est facile de voir que la condition d'un tel point (savoir d'un point dans lequel la tangente coupe la courbe en quatre points consécutifs) consiste en ce que $\partial^{3} \boldsymbol{U}$ contient $\partial \boldsymbol{U}$ comme facteur, ou, autrement dit, que l'équation $D^{3} \boldsymbol{U}=\mathbf{0}$ est identiquement vraie. On obtient ainsi dix conditions, qui se réduisent assez facilement à six; mais pour les réduire à une seule condition, il faut prendre la dérivée, avec le symbole $\boldsymbol{D}$ de la valeur donnée ci-dessus de $D^{2} U$. On doit cependant ne pas oublier que $D \cdot D^{2} U$, outre le terme $D^{3} U$, contient aussi des termes que l'on obtient en fésant opérer les symboles $\partial_{x}, \partial_{y}, \partial_{z}$ sur les quantités $L, M, N$ qui entrent dans $D^{2} U$. Car il est convenu que les symboles $\partial_{x}, \partial_{y}, \partial_{z}$ dans $D^{3} \boldsymbol{U}$, ne doivent pas affecter les lettres $\boldsymbol{L}, \boldsymbol{M}, \boldsymbol{N}$. Cejendant il est remarquable que dans le cas actuel, ces termes se détruisent entièrement. En effet, en les désignant par $\Omega$, on obtient:

$$
\begin{gathered}
\Omega=2 D \cdot[\boldsymbol{M} \gamma-\boldsymbol{N} \beta)\left(\boldsymbol{h} \partial_{L}+h \partial_{M}+g \partial_{N}\right)+(\boldsymbol{N} \alpha-\boldsymbol{L} \boldsymbol{\beta}) \cdot\left(\boldsymbol{h} \partial_{L}+b \partial_{M}+f \partial_{N}\right) \\
\left.+(\boldsymbol{L} \beta-\boldsymbol{M} \boldsymbol{\gamma})\left(\boldsymbol{g} \partial_{L}+f \partial_{M}+\boldsymbol{c} \partial_{N}\right)\right] \boldsymbol{D} \cdot \boldsymbol{U},
\end{gathered}
$$


ou il faut d'abord effectuer les opérations $\partial_{L}, \partial_{M}, \partial_{N}$ qui se rapportent à $\boldsymbol{D}$, et ensuite rapporter les $\partial_{x}, \partial_{y}, \partial_{z}$ à la fonction $\boldsymbol{U}$. Cela donne

$$
\begin{aligned}
& \boldsymbol{\Omega}=\mathbf{2} \cdot\left[\alpha\left(\boldsymbol{N} \partial_{y}-\boldsymbol{M} \partial_{z}\right)+\boldsymbol{\beta}\left(\boldsymbol{L} \partial_{z}-\boldsymbol{N} \partial_{y}\right)+\gamma\left(\boldsymbol{M} \partial_{x}-\boldsymbol{L} \partial_{y}\right)\right] \times \\
& \left\{\begin{array}{c}
\alpha^{2}\left[\boldsymbol{N}\left(\boldsymbol{f} \partial_{y}-\boldsymbol{b} \partial_{z}\right)-\boldsymbol{M}\left(\boldsymbol{c} \partial_{y}-\boldsymbol{f} \partial_{z}\right)\right] \\
+\beta^{2}\left[\boldsymbol{L}\left(\boldsymbol{g} \partial_{z}-\boldsymbol{c} \partial_{x}\right)-\boldsymbol{N}\left(\boldsymbol{a} \partial_{z}-g \partial_{x}\right)\right] \\
+\gamma^{2}\left[\boldsymbol{M}\left(\boldsymbol{h} \partial_{x}-a \partial_{y}\right)-\boldsymbol{L}\left(\boldsymbol{b} \partial_{x}-\boldsymbol{h} \partial_{y}\right)\right] \\
+\beta \gamma\left[-\boldsymbol{L}\left(\boldsymbol{h} \partial_{z}+g \partial_{y}-2 f \partial_{x}\right)+\boldsymbol{M}\left(\boldsymbol{a} \partial_{z}-g \partial_{x}\right)+\boldsymbol{N}\left(\boldsymbol{a} \partial_{y}-h \partial_{x}\right)\right] \\
+\gamma \alpha\left[-\boldsymbol{M}\left(\boldsymbol{f} \partial_{x}+h \partial_{z}-2 g \partial_{y}\right)+\boldsymbol{N}\left(b \partial_{x}-h \partial_{y}\right)+\boldsymbol{L}\left(\boldsymbol{b} \partial_{z}-f \partial_{y}\right)\right] \\
+\alpha \beta\left[-\boldsymbol{N}\left(g \partial_{y}+f \partial_{x}-2 h \partial_{z}\right)+\boldsymbol{L}\left(\boldsymbol{c} \partial_{y}-f \partial_{z}\right)+\boldsymbol{M}\left(\boldsymbol{c} \partial_{x}-g \partial_{z}\right)\right]
\end{array}\right\} \boldsymbol{U},
\end{aligned}
$$

où $\partial_{x}, \partial_{y}, \partial_{z}$ se rapportent seulement à $\boldsymbol{U}$. Or tous les termes de cette expression s'évanouissent. Par exemple le coefficient de $\alpha^{3}$ devient

$$
\left(\boldsymbol{N} \partial_{y}-\boldsymbol{M} \partial_{z}\right)\left[\mathbf{N}\left(f \partial_{y}-b \partial_{z}\right)-\boldsymbol{M}\left(\boldsymbol{c} \partial_{y}-f \partial_{z}\right)\right] \boldsymbol{U}
$$

$=\left[\boldsymbol{N}^{2}\left(f \partial_{y}^{2}-b \partial_{z} \partial_{y}\right)+M^{2}\left(c \partial_{y} \partial_{z}-f \partial_{z}^{2}\right)-\boldsymbol{M N}\left(\boldsymbol{c} \partial_{y}^{2}-f \partial_{\gamma} \partial_{z}+f \partial_{y} \partial_{z}-b \partial_{z}^{2}\right) U=0\right.$, et ainsi des autres termes; donc on a $\Omega=0$.

Donc, en transportant à l'autre côté de l'équation les termes qui contiennent $\boldsymbol{U}, \boldsymbol{D U}$ ou $\nabla \boldsymbol{U}$, on obtient cette formule très simple:

$$
(\boldsymbol{n}-1)^{2} \boldsymbol{D}^{3} \boldsymbol{U}=-(\boldsymbol{x}+\boldsymbol{\beta} \boldsymbol{y}+\boldsymbol{\gamma} \boldsymbol{z})^{2} \boldsymbol{D} \nabla \boldsymbol{U},
$$

où la seule condition d'un point d'osculation (en ayant égard à l'équation $\nabla \boldsymbol{U}=0$ ) se réduit à $\boldsymbol{D} \nabla \boldsymbol{U}=0$. Savoir les dérivées $\partial_{x} \nabla \boldsymbol{U}, \partial_{y} \nabla \boldsymbol{U}, \partial_{z} \nabla \boldsymbol{U}$ doivent être proportionnelles à $\partial_{x} \boldsymbol{U}, \partial_{y} \boldsymbol{U}, \partial_{z} \boldsymbol{U}$ (ce qui équivaut à une seule condition, en vertu de $\boldsymbol{U}=0, \nabla \boldsymbol{U}=0$; comme on le voit facilement). Cela donne le théorème suivant.

Théorène. „Dans le cas d'un point d'osculation, les deux courbes $\boldsymbol{U}=0, \nabla \boldsymbol{U}=0$ se touchent."

Il n'y a presque pas de doute que la derivée $\nabla \nabla U$ ne se réduise toujours à la forme $\boldsymbol{R} \cdot \boldsymbol{U}+\boldsymbol{S} \cdot \nabla \boldsymbol{U}$. En effet, M. Hesse l'à démontré pour les fonctions de trois variables et du troisième degré, et moi, je l'ai vérifié pour les fonctions de deux variables d'un dégré quelconque. Cela étant, les points d'inflexion de la courbo $\nabla \boldsymbol{U}=0$ sont situés aux points d'intersection avec $U=0$, et au cas ou les deux courbes se touchent, ce point de contact doit être considéré comme la réunion de trois points d'intersection: donc

„Tout point d'osculation peut être envisagé comme point de réunion de trois points d'inflexion:"

Nous démontrerons encore d'une manière conforme à celle dont nous avons trouvé l'expression de $D^{2} U$, l'expression qui vient d'ètre donnée pour $D^{3} U$ 
de la formule

$$
\varrho^{2}(123)^{2}=\left(T_{1}(A 23)+T_{2}(A 31)+T_{3}(A 12)\right)^{2} .
$$

En multipliant les deux membres par $(\boldsymbol{A 1 4})+(\boldsymbol{A} 24)+(\boldsymbol{A} 34)$, le terme à gauche peut être présenté sous la forme $\varphi^{2} \cdot(A \theta 4) \cdot(123)^{2}$, où $\partial_{x_{\theta}}, \partial_{\gamma_{\theta}}, \partial_{\tilde{z}_{\theta}}$ se rapportent à tous les systèmes de variables. En y appliquant le produit $\boldsymbol{U}_{1} \boldsymbol{U}_{2} \boldsymbol{U}_{3} \boldsymbol{U}_{+}$ (les variables identiques après les différentiations), on obtiendra $6 \varrho^{2}(\boldsymbol{A} \theta 4) U_{4} \cdot \nabla U$ $=-6 \cdot \boldsymbol{D} \nabla \boldsymbol{U} \cdot \rho^{2}$. Pour la droite de l'équation on a d'abord trois termes comme $\boldsymbol{T}_{1}^{2} \cdot(\boldsymbol{A} 14)(\boldsymbol{A} 23)^{2} \cdot U_{1} U_{2} U_{3} U_{4}$, lesquels se détruisent évidemment, à cause de $(\boldsymbol{A} 14) \cdot U_{1} U_{4}=0$; puis six termes de la forme $(\boldsymbol{A} 14)(\boldsymbol{A} 23)(\boldsymbol{A} 31) U_{1} U_{2} U_{3} U_{4}$, qui se détruisent aussi, puisqu'en changeant 1,3 et 2,4 , le terme change de signe; puis trois termes comme $\boldsymbol{T}_{1}^{\prime 2} \cdot((\boldsymbol{A} 24)+(\boldsymbol{A} 34))(\boldsymbol{A} 23)^{2} \cdot \boldsymbol{U}_{1} \boldsymbol{U}_{2} \boldsymbol{U}_{3} \boldsymbol{U}_{4}$ etc. savoir $\boldsymbol{T}_{1}^{2} \cdot \boldsymbol{U}_{1}((\boldsymbol{A} 24)+(\boldsymbol{A 3 4}))(\boldsymbol{A} 23)^{2} \cdot U_{2} U_{3} U_{4}=-2 n(n-1) \boldsymbol{U} \cdot \boldsymbol{D} \Psi$; c'està-dire tous ces termes sont $6 n(n-1) U \cdot D \Psi$; enfin trois termes de la forme $2 T_{1} T_{2}(A 23)(A 31)(A 34) U_{1} U_{2} U_{3} U_{4}=2(n-1)^{2} \cdot D^{3} U$, ou, tous pris ensemble, $6(n-1)^{2} D^{3} U$. Donc, en supprimant le terme $-6 n(n-1) U \cdot D \Psi$, à cause de $U=0$, on obtient la mème équation que ci-dessus, savoir:

$$
(n-1)^{2} D^{3} \boldsymbol{U}=-(\alpha \boldsymbol{x}+\beta y+\gamma z)^{2} \boldsymbol{D} \nabla \boldsymbol{U} \text {. }
$$

On pourrait croire qu'il y a une équation analogue $(n-1)^{2} D^{+} U=$ $-(\alpha x+\beta y+\gamma z)^{2} D^{2} \nabla U$ pour les derrivées du quatrième degré, mais cela n'est pas. En effet, il est facile de voir que pour un point d'osculation, $D^{2} \nabla \boldsymbol{U}$ se réduit à $\nabla \nabla \boldsymbol{U}$, à un facteur près, c'est-à-dire de $\boldsymbol{D}^{2} \nabla \boldsymbol{U}=\mathbf{0}$, puisque $\nabla \nabla \boldsymbol{U}$ s'évanouit aux points d'osculation: donc on aurait généralement pour un point d'osculation $D^{4} U=0$; mais cela est seulement le caractère des points d'osculation d'un plus haut degré, savoir de ceux où la tangente rencontre la courbe en cinq points consécutifs. Pour le quatrième degré le problème devient trop compliqué pour être traité de cette manière.

Cambridge $9^{\text {iène }}$ mai 1846. 